\title{
Characterising the Dynamo in a Radiatively Inefficient Accretion Flow
}

\author{
Prasun Dhang ${ }^{1,3,4},{ }^{\star}$ Abhijit Bendre ${ }^{2}, \dagger$ Prateek Sharma $^{1}, \ddagger$ Kandaswamy Subramanian ${ }^{2} \S$ \\ ${ }^{1}$ Department of Physics and Joint Astronomy Programme, Indian Institute of Science, Bangalore, INDIA 560012 \\ 2 IUCAA, Post Bag 4, Ganeshkhind, Pune 411 007, INDIA \\ ${ }^{3}$ Institute for Advanced Study, Tsinghua University, Beijing 100084, China \\ ${ }^{4}$ Department of Astronomy, Tsinghua University, Beijing 100084, China
}

\begin{abstract}
We explore the MRI driven dynamo in a radiatively inefficient accretion flow (RIAF) using the mean field dynamo paradigm. Using singular value decomposition (SVD) we obtain the least squares fitting dynamo coefficients $\alpha$ and $\gamma$ by comparing the time series of the turbulent electromotive force and the mean magnetic field. Our study is the first one to show the poloidal distribution of these dynamo coefficients in global accretion flow simulations. Surprisingly, we obtain a high value of the turbulent pumping coefficient $\gamma$ which transports the mean magnetic flux radially outward. This would have implications for the launching of magnetised jets which are produced efficiently in presence a large-scale poloidal magnetic field close to the compact object. We present a scenario of a truncated disc beyond the RIAF where a large scale dynamogenerated poloidal magnetic field can aid jet-launching close to the black hole. Magnitude of all the calculated coefficients decreases with radius. Meridional variations of $\alpha_{\phi \phi}$, responsible for toroidal to poloidal field conversion, is very similar to that found in shearing box simulations using the 'test field' (TF) method. By estimating the relative importance of $\alpha$-effect and shear, we conclude that the MRI driven large-scale dynamo, which operates at high latitudes beyond a disc scale height, is essentially of the $\alpha-\Omega$ type.
\end{abstract}

Key words: accretion,accretion discs - dynamo - instabilities - magnetic fields - MHD turbulence - methods: numerical.

\section{INTRODUCTION}

Angular momentum transport in a completely ionized rotationallysupported accretion disc (such as in a black hole binary [BHB]) is supposed to be mediated by a weak field instability; namely, the Magneto-rotational instability (MRI; Velikhov 1959; Chandrasekhar 1960; Balbus \& Hawley 1991). Although linear MRI guarantees outward angular momentum transport, its saturation determines different accretion properties such as accretion rate and luminosity. With the increase in computational capabilities, it has been possible in last two decades to study saturation of the MRI using both local (shearing box) and global simulations with increasing resolution. Previous local (Brandenburg et al. 1995; Hawley et al. 1996; Davis et al. 2010; Gressel \& Pessah 2015; Bhat et al. 2016) and global (Flock et al. 2012; Hawley et al. 2013; Parkin \& Bicknell 2013; Suzuki \& Inutsuka 2014; Jiang et al. 2014; Hogg \& Reynolds 2018) studies show that an MRI driven dynamo can sustain magnetic fields in saturation, overcoming the dissipative effects (for a

\footnotetext{
* E-mail:prasundhang@gmail.com

† abbendre@gmail.com

¥ prateek@iisc.ac.in

$\S$ kandu@iucaa.in
}

review see Blackman \& Nauman (2015)). They also show that the MRI driven dynamo generates large scale magnetic fields. Large scale magnetic fields are not only the necessary ingredient to produce outflows/jets (Blandford \& Znajek 1977; Blandford \& Payne 1982), they are also important in determining the level of angular momentum transport (Johansen \& Levin 2008; Bai \& Stone 2013) in accretion discs.

Till date, most studies focus on dynamo action in the standard disc either using a local or a global approach. All the global (Arlt \& Rüdiger 2001; Flock et al. 2012; Hogg \& Reynolds 2018) and a few local shearing-box (Brandenburg et al. 1995; Davis et al. 2010) simulations use a very simple mean field closure (equation 23) to characterise the dynamo coefficients in shearing box simulations. A few studies (Brandenburg 2008; Gressel 2010; Gressel \& Pessah 2015) consider a more complicated closure encapsulating the anisotropic nature of MHD turbulence. Using state of the art test field (TF) method (Schrinner et al. 2007; Brandenburg 2009), these studies determine turbulent dynamo coefficients for the MRI driven dynamo.

In this paper, we wish to characterise the mean field dynamo in a hot, optically thin, geometrically thick radiatively inefficient accretion flow (RIAF; Narayan \& Yi 1994; Blandford \& Begelman 
1999; Narayan et al. 2000; Quataert \& Gruzinov 2000; Yuan et al. 2012; Yuan \& Narayan 2014). We use the model 'M-2P' described in Dhang \& Sharma (2019) to calculate the dynamo coefficients. Most of the previous studies determining the dynamo coefficients in local shearing box simulations use the TF method. We take an alternate approach using singular value decomposition (SVD) method (Racine et al. 2011; Simard et al. 2016; Bendre et al. 2019). In this method, we essentially solve the problem of a least-square minimisation by fitting the time series of turbulent EMF with that of the mean magnetic fields. The advantage of SVD method is that we can post-process the simulation data, while the TF method requires to solve additional equations for the passive fluctuating fields generated by large scale test fields.

It is thought that there is a close connection between the jet and the existence of a RIAF close to the black hole (Esin et al. 1997; Fender et al. 1999). Most of the previous studies investigating the disc-jet coupling assume the presence of a large-scale magnetic field (Tchekhovskoy et al. 2011; McKinney et al. 2012; Penna et al. 2013). However, the source of this coherent large-scale magnetic fields is still an open question. The two possible generation mechanisms are (i) advection of field from the outer standard thin disc (BisnovatyiKogan \& Ruzmaikin 1974, 1976) and (ii) in-situ generation of the field by dynamo action (Brandenburg et al. 1995; Hawley et al. 1996). In a classical turbulent thin $\operatorname{disc}(H / R \ll 1)$, the advection timescale is comparable to the turbulent diffusion timescale (Lubow et al. 1994; Cao 2018). However, a few studies propose that in the hot tenuous coronal region (where radial velocity is comparatively higher compared to that in the mid-plane) above and below the disc mid-plane, field can be dragged inward efficiently (Guilet \& Ogilvie 2012, 2013). There are some recent studies (Hogg \& Reynolds 2018; Liska et al. 2018), including ours (Dhang \& Sharma 2019), which investigate dynamo action in RIAFs. Large scale dynamo action is found to be weak in RIAFs and only confined beyond the disc scale height, within which a turbulent small-scale dynamo dominates.

By using the SVD method, we obtain the distribution of turbulent dynamo coefficients in the poloidal $(r, \theta)$ plane for a RIAF. We emphasise the previously unnoticed effect of strong radial turbulent pumping (or what is known as the $\gamma$-effect), which transports largescale magnetic fields radially outward. Presence of this $\gamma$-effect makes it harder for the large-scale magnetic field to be advected towards the black hole even in a weakly magnetized RIAF (like model 'M-2P'). We propose a possible scenario for flux accumulation near the black hole in the truncated disc model (Esin et al. 1997) which is favourable for jet formation in the low/hard state of a BHB.

The paper is organised as follows. In section 2 we briefly discuss the details of the simulation which we analyse. The mean field formalism and the SVD method are discussed in section 3. We describe the results in section 4. A discussion of the results and their astrophysical implications is presented in section 5. Finally, we summarise the key findings of the paper in section 6 .

\section{THE SIMULATION DETAILS}

We retrieve the dynamo coefficients for the MRI driven dynamo in a RIAF. We use the model 'M-2P' described in Dhang \& Sharma (2019). To summarise, we solve the Newtonian ideal MHD equations of motion of the magnetised gas around a non-spinning black hole using the PLUTO code (Mignone et al. 2007). Considering an ideal equation of state with adiabatic index $\Gamma=5 / 3$, we solve the equations in spherical coordinates $(r, \theta, \phi)$ with the computational domain spanning over $r \in[4,140], \theta \in[0.02, \pi-0.02], \phi \in$
$[0,2 \pi]$ with a resolution $N_{r} \times N_{\theta} \times N_{\phi}=368 \times 192 \times 512$. Most of the grids are employed in the region $r \in[4,45], \theta \in[\pi / 3,2 \pi / 3]$, $\phi \in[0,2 \pi]$ where bulk of the accreting gas is present (for a detailed description see Table 1 and section 2.3 of Dhang \& Sharma (2019)).

In the Newtonian regime, to mimic the general relativistic effects close to a black hole, we use the pseudo-Newtonian potential (Paczyńsky \& Wiita 1980) $\Phi=G M /\left(r-2 r_{g}\right)$, where $r_{g}=$ $G M / c^{2}$ is the gravitational radius, $M$ and $c$ are the mass of the accreting black hole and the speed of light in vacuum respectively. In the code, we assume $G M=c=1$ to work in dimensionless units. As a result, all the length scales and velocities are expressed in the units of $r_{g}$ and $c$ respectively. Unless stated otherwise, time scales are expressed in terms of the number of orbits a test particle would do at the inner most stable circular orbit (ISCO), and is given by

$$
N_{\mathrm{ISCO}}=\frac{t_{\mathrm{sim}}}{T_{\mathrm{ISCO}}}
$$

where the simulation time is $t_{\mathrm{sim}}$, the orbital period at ISCO, $T_{\mathrm{ISCO}}=2 \pi r_{\mathrm{ISCO}}^{1 / 2}\left(r_{\mathrm{ISCO}}-2\right)=61.56 r_{g} / c$ and we use $r_{\mathrm{ISCO}}=6 r_{g}$ for a Schwarzschild black hole.

We initialise a constant angular momentum torus (Papaloizou \& Pringle 1984), embedded in a non-rotating, low-density hydrostatic medium. A poloidal magnetic field of plasma beta $\beta_{\text {ini }}=890$ is initially threading the equilibrium torus and is parallel to the density contours.

For the extraction of dynamo coefficients, we use the time series of mean magnetic fields and EMFs in the quasi-steady state with a span $t_{\operatorname{sim}} \in[250,630]$ and data dumping interval $\Delta t=$ $t_{i+1}-t_{i}=1.61 N_{\text {ISCO }}$. Also, we only cover the radial range $r \in[10,50] r_{g}$ which is roughly in a statistically stationary state, given that the inflow equilibrium radius is $r_{\mathrm{eq}}=t_{\mathrm{visc}} v_{r} \approx 40 r_{g}$ (see section 5.7 of Dhang \& Sharma (2019)). Here, $t_{\mathrm{visc}}$ and $v_{r}$ are the viscous time and radial velocity respectively.

\section{THE MEAN FIELD DYNAMO}

To understand the evolution of large-scale magnetic fields in the simulations, we employ the standard mean-field dynamo formalism. Its mathematical formulation of relies upon splitting of the flow variables, velocity $\mathbf{v}$ and magnetic field $\mathbf{B}$ as a sum of large scale or mean components (denoted by over-bars, $\overline{\mathbf{v}}$ and $\overline{\mathbf{B}}$ ) and small-scale or fluctuating components (denoted by primes $\mathbf{v}^{\prime}$ and $\mathbf{B}^{\prime}$ ), where the mean is defined over a suitable averaging domain that usually satisfies Reynolds rules. We thus define mean of a quantity $q$ by integrating over the azimuthal domain as,

$$
\bar{q}(r, \theta, t)=\frac{1}{2 \pi} \int_{0}^{2 \pi} q(r, \theta, \phi, t)
$$

and consequently the fluctuations as

$$
q^{\prime}(r, \theta, \phi, t)=q(r, \theta, \phi, t)-\bar{q}(r, \theta, t) .
$$

Time evolution of the mean magnetic field is then governed by averaged induction equation,

$$
\frac{\partial \overline{\mathbf{B}}}{\partial t}=\nabla \times\left[\overline{\mathbf{v}} \times \overline{\mathbf{B}}+\overline{\mathbf{v}^{\prime} \times \mathbf{B}^{\prime}}-\eta \nabla \times \overline{\mathbf{B}}\right]
$$

where $\eta$ is the microscopic diffusivity. The terms within the bracket on the right-hand side describe the effects of mean and fluctuating fields and flows on its evolution. The effect of turbulence on the 
mean field evolution is captured through the mean electromotive force (EMF),

$$
\overline{\mathcal{E}}=\overline{\mathbf{v}^{\prime} \times \mathbf{B}^{\prime}} .
$$

Motivated by Second Order Correlation Approximation (SOCA) (Moffatt 1978; Krause \& Rädler 1980), the EMF is modelled as linear function of mean field and its covariant derivatives ignoring any higher order derivatives. In a locally Cartesian co-ordinate system we have,

$$
\overline{\mathcal{E}}_{i}=a_{i j} \bar{B}_{j}+b_{i j k} \frac{\partial \bar{B}_{j}}{\partial x_{k}} .
$$

Here the tensorial coefficients $a_{i j}$ and $b_{i j k}$ are the functions of statistical correlation among fluctuating parts of velocity and magnetic fields. Further, a is a rank two tensor which acts as a source term in equation 4 , and $\mathbf{b}$, a rank three tensor is mainly responsible for diffusion of mean magnetic field. By further decomposing these tensors into their symmetric and anti-symmetric parts, equation 6 can be rewritten as,

$$
\begin{array}{r}
\overline{\mathcal{E}}_{i}=\alpha_{i j} \bar{B}_{j}+(\boldsymbol{\gamma} \times \overline{\mathbf{B}})_{i}-\eta_{i j}(\nabla \times \overline{\mathbf{B}})_{j} \\
-[\boldsymbol{\Delta} \times(\nabla \times \overline{\mathbf{B}})]_{i}-\kappa_{i j k} \frac{\partial \overline{\mathrm{B}}_{j}}{\partial x_{k}}
\end{array}
$$

(See eg. Schrinner et al. 2007, and references therein. ) Here the dynamo coefficients $\alpha, \gamma, \eta, \Delta$ and $\kappa$ describe the various turbulence properties, eg.

$$
\alpha_{i j}=\frac{1}{2}\left(a_{i j}+a_{j i}\right), \text { and } \gamma_{k}=\frac{1}{2} \epsilon_{k i j} a_{i j}
$$

describe the classical $\alpha$-effect and turbulent pumping respectively. It can be explicitly seen from equation 7 and equation 4 that $\gamma$ adds to the mean velocity $\overline{\mathbf{v}}$ in advecting the mean magnetic field. The tensor $\eta_{i j}$ represents the diagonal and off-diagonal parts of anisotropic turbulent diffusivity, while the coefficient $\Delta$ encapsulates a dynamo generating term first identified by Rädler (1969). In this analysis however we have neglected the contribution of tensor $\mathbf{b}$, since it had no significant impact on the determination of EMF.

\subsection{Estimating Dynamo Coefficients Using the SVD Method}

The dynamo coefficients in equation 7 express the effects of MHD turbulence on the evolution of the mean field. It is therefore of interest to determine these coefficients for the direct simulations we have performed. To explicitly state the problem we first express the $i^{\text {th }}$ component EMF using equation 6 and ignore the higher order terms involving tensor $\mathbf{b}$ in the expansion (similar to Racine et al. 2011) as,

$$
\overline{\mathcal{E}}_{i}^{M}=a_{i j} \bar{B}_{j}
$$

where $i, j \in(r, \theta, \phi)$, and the pseudo-tensor $a_{i j}$ relates to the direct dynamo coefficients $\alpha_{i j}$ and $\gamma_{i}$ in equation 8 through following relations,

$$
\begin{array}{cll}
\alpha_{r r} & = & a_{r r}, \\
\alpha_{\theta \theta} & = & a_{\theta \theta}, \\
\alpha_{\phi \phi} & = & a_{\phi \phi}, \\
\alpha_{r \theta}=\alpha_{\theta r} & = & \frac{1}{2}\left(a_{r \theta}+a_{\theta r}\right) \\
\alpha_{r \phi}=\alpha_{\phi r} & =\frac{1}{2}\left(a_{r \phi}+a_{\phi r}\right), \\
\alpha_{\theta \phi}=\alpha_{\phi \theta} & =\frac{1}{2}\left(a_{\theta \phi}+a_{\phi \theta}\right) .
\end{array}
$$

$$
\begin{aligned}
\gamma_{r} & =\frac{1}{2}\left(a_{\theta \phi}-a_{\phi \theta}\right), \\
\gamma_{\theta} & =\frac{1}{2}\left(a_{\phi r}-a_{r \phi}\right), \\
\gamma_{\phi} & =\frac{1}{2}\left(a_{r \theta}-a_{\theta r}\right) .
\end{aligned}
$$

The problem then is one of determining $a_{i j}$ and from the data of DNS, at each point in $(r, \theta)$. Note here that we have ignored the contribution of tensor $\mathbf{b}$ as it was found to have no statistically significant effect on the determination of EMF, as discussed in the later sections. The expressions for the complete set of dynamo coefficients is given in Appendix A (see also Schrinner et al. 2007, and references therein). Due to insufficient number of equations than are required to have a unique solution to equation 9, we treat this problem as the one of time series analysis. In particular, we adopt the standard singular value decomposition method to minimise the least-squares of the time series formed from the residuals of equation 9 at each $(r, \theta)$ point. We have adopted this method from Racine et al. (2011) and Simard et al. (2016) and the details are discussed below.

At each point in $(r, \theta)$ plane, the time series of EMF components $\overline{\mathcal{E}}_{i}$ and mean magnetic field $\bar{B}_{i}$ are extracted from the DNS. Elements of these time series are treated as independent data points, and used to determine the values of $a_{i j}$ at $(r, \theta)$. This is achieved by determining the set of coefficients that minimise the least-square sums of following residual vector components;

$$
R_{i}=\overline{\mathcal{E}}_{i}-a_{i j} \bar{B}_{j},
$$

by using the SVD scheme.

We first construct the 'design matrix', $\mathcal{A}$ at each fixed $(r, \theta)$ point which we define as,

$$
\mathcal{A}=\left(\begin{array}{ccc}
\bar{B}_{r}\left(t_{1}\right) & \bar{B}_{\theta}\left(t_{1}\right) & \bar{B}_{\phi}\left(t_{1}\right) \\
\bar{B}_{r}\left(t_{2}\right) & \bar{B}_{\theta}\left(t_{2}\right) & \bar{B}_{\phi}\left(t_{2}\right) \\
\vdots & \vdots & \vdots \\
\bar{B}_{r}\left(t_{N}\right) & \bar{B}_{\theta}\left(t_{N}\right) & \bar{B}_{\phi}\left(t_{N}\right)
\end{array}\right)
$$

$\mathcal{A}$ is determined directly from the DNS data. The number of rows $N$, indicates the length of these time series, $\left(t_{1}, t_{2}, \ldots, t_{N}\right)$. Similarly we define the data matrix $\mathcal{Y}$ and the parameter matrix $\mathcal{X}$ (also at $(r, \theta))$ as,

$$
\mathcal{Y}=\left(\begin{array}{ccc}
\overline{\mathcal{E}}_{r}\left(t_{1}\right) & \overline{\mathcal{E}}_{\theta}\left(t_{1}\right) & \overline{\mathcal{E}}_{\phi}\left(t_{1}\right) \\
\overline{\mathcal{E}}_{r}\left(t_{2}\right) & \overline{\mathcal{E}}_{\theta}\left(t_{2}\right) & \overline{\mathcal{E}}_{\phi}\left(t_{2}\right) \\
\vdots & \vdots & \vdots \\
\overline{\mathcal{E}}_{r}\left(t_{N}\right) & \overline{\mathcal{E}}_{\theta}\left(t_{N}\right) & \overline{\mathcal{E}}_{\phi}\left(t_{N}\right)
\end{array}\right),
$$

and

$$
\mathcal{X}=\left(\begin{array}{ccc}
a_{r r} & a_{\theta r} & a_{\phi r} \\
a_{r \theta} & a_{\theta \theta} & a_{\phi \theta} \\
a_{r \phi} & a_{\theta \phi} & a_{\phi \phi}
\end{array}\right) .
$$

Noting that matrices $\mathcal{X}, \mathcal{Y}$ and $\mathcal{A}$ are of dimensions $3 \times 3, N \times 3$ and $N \times 3$ respectively, equation 9 can be written as,

$$
\mathcal{Y}(r, \theta)=\mathcal{A}(r, \theta) \mathcal{X}(r, \theta)+\hat{\mathcal{N}}(r, \theta) .
$$

The additional term $\hat{\mathcal{N}}$ on the right hand side is the noise matrix with same dimensions as $\mathcal{Y}$. Columns of $\hat{\mathcal{N}}$ represent the level of additive noise in the components of $\overline{\mathcal{E}}$ while the rows represent the additive noise in the respective EMF component at a fixed time. With this arrangement equation 16 now represents an over-determined 
system of equations wherein the constancy of the elements of $\mathcal{X}$ is implicitly assumed for $t_{1}<t<t_{N}$. Such an assumption appears reasonable in the saturated steady state of the dynamo. The least squared solution $(\hat{\mathcal{X}})$ to this system is found by seeking to minimise the following two norm for $i^{\text {th }}$ columns of $\mathcal{Y}$ and $\mathcal{X}$,

$$
\chi_{i}^{2}(r, \theta)=\frac{1}{N} \sum_{n=1}^{N}\left[\frac{\left(\mathbf{y}_{i}\left(r, \theta, t_{n}\right)-\mathcal{A} \mathbf{x}_{i}\left(r, \theta, t_{n}\right)\right)^{\top}}{\sigma_{i}}\right]^{2} .
$$

Here the vectors $\mathbf{y}_{i}$ and $\mathbf{x}_{i}$ are the $i^{\text {th }}$ columns of $\mathcal{Y}$ and $\mathcal{X}$ respectively. While $\sigma_{i}$ is the variance associated with $i^{\text {th }}$ column of the noise matrix $\hat{\mathcal{N}}$ (which we will determine from the best fit $\hat{\mathbf{x}}_{i}$ itself below). The method that we use here to seek the least square solution $\hat{\mathcal{X}}$, relies upon the unique SVD decomposition of $\mathcal{A}$;

$$
\mathcal{A}=\mathbf{U} \mathbf{w} \mathbf{V}^{T}
$$

where the matrices $\mathbf{U}$ and $\mathbf{V}$ are of dimensions $N \times 3$ and $3 \times$ 3 respectively and are orthonormal, while the singular matrix $\mathbf{w}$ is $3 \times 3$ diagonal matrix. For this decomposition we follow the algorithm described in Press et al. (1992). It is to be noted here that matrices $\mathbf{A}, \mathbf{U}, \mathbf{V}$ and $\mathbf{w}$ are functions of $r$ and $\theta$. We avoid the explicit mention of this fact hereafter, unless required. $\hat{\mathcal{X}}$ can then be expressed in terms of $\mathbf{U}, \mathbf{V}$ and $\mathbf{w}$ simply as (similar to Mandel 1982),

$$
\hat{\mathbf{x}}_{j}=\mathbf{V} \mathbf{w}^{-1} \mathbf{U}^{T} \mathbf{y}_{j} .
$$

We recall that here the index ' $j$ ' denotes the $j$ 'th column of either $\hat{\mathcal{X}}$ or $\mathcal{Y}$. Variances associated with each element of $\hat{\mathbf{x}}_{j}$ do depend upon the column index $j$ (through the $\sigma_{j}$ in equation 17) as,

$$
\operatorname{Var}\left(\left[\hat{\mathbf{x}}_{j}\right]_{i}\right)=\sum_{l}\left[\frac{\mathbf{V}_{i l}}{\mathbf{w}_{l l}}\right]^{2} \sigma_{j}^{2}
$$

where the noise component $\sigma_{j}$ is calculated from the SVD fit simply as,

$$
\sigma_{j}^{2}=\frac{1}{N}\left(\mathbf{y}_{j}-\mathcal{A} \hat{\mathbf{x}}_{j}\right)^{\top}\left(\mathbf{y}_{j}-\mathcal{A} \hat{\mathbf{x}}_{j}\right)
$$

\section{RESULTS}

Saturation of the MRI in the non-linear regime maintaining a finite amplitude for the magnetic field indicates an underlying dynamo action. A distinguishing feature of the dynamo action in a geometrically thick RIAF is the presence of an intermittent dynamo cycle (Hogg \& Reynolds 2018; Dhang \& Sharma 2019; Liska et al. 2018). Irregularity in the dynamo cycle can readily be explained using the mean field dynamo theory (for a review see Brandenburg \& Subramanian 2005). Slightly sub-Keplerian nature of the angular velocity leads to the intermittency (Nauman \& Blackman 2015; Gressel \& Pessah 2015; Dhang \& Sharma 2019).

\subsection{Butterfly diagrams for the mean fields}

Fig 1 shows the variation of the mean magnetic fields with latitude $\theta_{l}=90^{\circ}-\theta$ and time (popularly known as the 'Butterfly diagram') at a radius $r=20$ for a geometrically thick $(H / R \sim 0.4)$ RIAF (model 'M-2P' in Dhang \& Sharma 2019). Top, middle and bottom panels of Fig. 1 show the spatio-temporal variation of radial $\left(\bar{B}_{r}\right)$, meridional $\left(\bar{B}_{\theta}\right)$ and toroidal $\left(\bar{B}_{\phi}\right)$ mean magnetic fields respectively. While $\bar{B}_{\phi}$ and $\bar{B}_{r}$ are the strongest components, strength of $\bar{B}_{\theta}$ is much smaller. It is also evident from Fig. 1 that mean fields
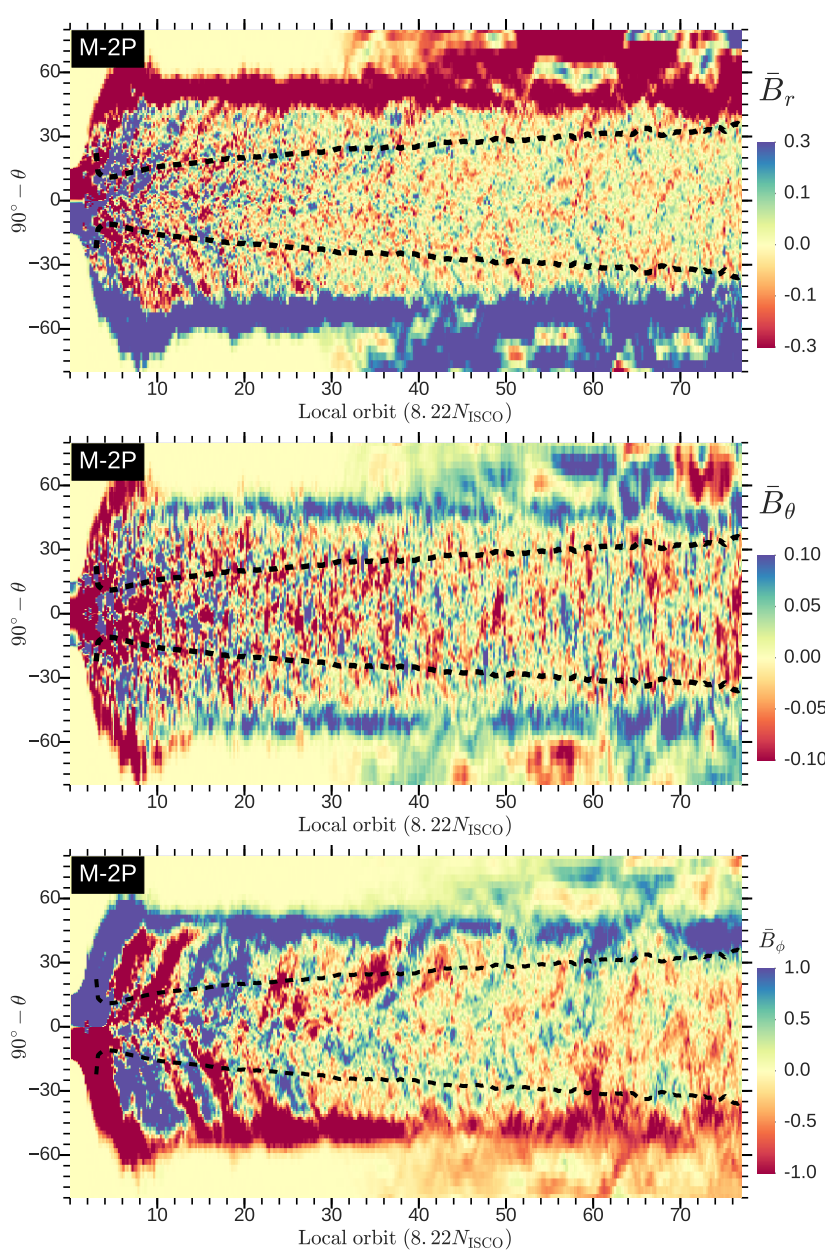

Figure 1. Spatio-temporal variation of radial (top panel), meridional (middle panel) and toroidal (bottom panel) components of mean magnetic fields at a radius $r=20$. Time is expressed in units of local orbit at $r=20$. Black dashed lines indicate one scale-height in the northern and southern hemispheres. Mean fields are stronger at larger latitudes on both the hemispheres, where large scale dynamo operates. Equatorial regions, where fluctuation dynamo dominates, have smaller mean fields.

are stronger at higher latitudes, where stratification becomes important and large scale dynamo operates. On the other hand, at lower latitudes around the mid-plane, fluctuation dynamo dominates and mean fields are vanishingly small. It should also be mentioned that the contribution to the Maxwell stress from the mean fields are larger where mean fields are predominant, while close to the midplane the Maxwell stress is mainly due to the correlation between the fluctuating components. For a detailed discussion on the two dynamo mechanisms in RIAFs see Fig. 26 and Section 7.4 in Dhang \& Sharma (2019).

\subsection{Spatial variation of the dynamo coefficients}

In an accretion flow, due to the presence of a strong shear, poloidal fields $\left(B_{r}\right.$ and $\left.B_{\theta}\right)$ are readily converted into toroidal fields $\left(B_{\phi}\right)$. The generation of poloidal fields is mainly attributed to the twisting of toroidal magnetic fields by helical turbulence, known as the classical $\alpha$-effect. A simple mean field closure (equation 23) neglecting $\gamma$, turbulent diffusion and off-diagonal terms of $\alpha_{i j}$ fails to provide an ordered $\alpha$ coefficient for the dynamo action in RIAFs (see Fig. 
25 of Dhang \& Sharma (2019)). In the current work, we use a more general closure (equation 9) for mean fields and EMFs.

\subsubsection{Meridional variation}

Fig. 2 shows the meridional variation of the nine dynamo coefficients at three different radii $r=15,20,35$ recovered using the SVD method. Top three panels in Fig. 2 show the variation of diagonal components of $\alpha_{i j}$ with latitude $\theta_{l}=90^{\circ}-\theta$. While $\alpha_{r r}$ and $\alpha_{\phi \phi}$ are ordered and antisymmetric about the mid-plane, $\alpha_{\theta \theta}$ shows less coherent structure and is symmetric about the mid-plane. Out of these three diagonal components, the most important one for the large-scale dynamo is the $\alpha_{\phi \phi}$, which is responsible for the production of poloidal fields $B_{r}$ and $B_{\theta}$ out of the toroidal field $B_{\phi}$. In the northern hemisphere (NH) $\alpha_{\phi \phi}$ is negative at lower latitude and becomes positive at higher latitude. An opposite trend is seen in southern hemisphere ( $\mathrm{SH})$. This antisymmetric behaviour of $\alpha_{\phi \phi}$ is expected from the naive picture of helical turbulence, where Coriolis forces break parity and the symmetry about the equator. The pattern in meridional variation of $\alpha_{\phi \phi}$ roughly matches with those found in local shearing box simulations (Brandenburg 2008; Gressel 2010). However, the result differs from some of the previous global simulations (Arlt \& Rüdiger 2001; Flock et al. 2012). We will discuss the sign of $\alpha_{\phi \phi}$ in different studies of MRI driven dynamo in detail in section 5.1.

Middle and bottom three panels in Fig. 2 show the meridional variation of symmetric $\left(\alpha_{r \theta}, \alpha_{r \phi}, \alpha_{\theta \phi}\right)$ and antisymmetric $\left(\gamma_{r}\right.$, $\gamma_{\theta}, \gamma_{\phi}$ ) parts of the off-diagonal components of a-tensor. While $\alpha_{r \phi}$ and its corresponding anti-symmetric component $\gamma_{\theta}$ are antisymmetric about the mid-plane, other components show a symmetric behaviour. Among the non-diagonal components, the $\gamma$ 's, which represent turbulent pumping, are of particular interest. The coefficients $\gamma$ represent the transport of the mean fields from a turbulent region to a more laminar region - a phenomenon of turbulent diamagnetism. Positive sign of radial component $\gamma_{r}$ at all latitudes implies a transport of mean fields from more turbulent region (close to the $\mathrm{BH}$ ) to a comparatively less turbulent region (away from the $\mathrm{BH})$. Similarly, the negative (positive) sign of $\gamma_{\theta}$ in the $\mathrm{NH}(\mathrm{SH})$ describes the pumping of mean fields from turbulent equatorial region to the more laminar coronal region. The coefficient $\gamma_{\phi}$ arises because of the non-alignment of angular velocity and gradient in total (magnetic + kinetic) specific turbulent energy (see equation 10.59 in Brandenburg \& Subramanian (2005)).

\subsubsection{Radial variation}

In Fig. 2, we see a clear pattern, smaller the radius, larger the value of the dynamo coefficients. To get a better picture of this behaviour, we look at variation of the dynamo coefficients at high latitudes (where large-scale dynamo dominates) with radius in Fig. 3. We take four meridional cuts - two in the $\mathrm{NH}$ (with $\theta_{l}=50^{\circ}, 65^{\circ}$ ), and two in the $\mathrm{SH}\left(\theta_{l}=-50^{\circ},-65^{\circ}\right)$. Selection of such meridional cuts helps us comprehend the radial variation as well the symmetry of the dynamo coefficients about the mid-plane.

Top, middle and bottom panels of Fig. 3 show the radial variation of diagonal, symmetric and antisymmetric components of the a tensor respectively. Among the diagonal components, both $\alpha_{r r}$ and $\alpha_{\phi \phi}$ show a coherent antisymmetric behaviour at all radii (e.g. compare black and magenta lines representing dynamo coefficients at $\theta_{l}=+65^{\circ}$ and $\theta_{l}=-65^{\circ}$ respectively). The symmetric nature of $\alpha_{\theta \theta}$ as we describe in section 4.2.1 is not prevalent at all radii. Like $\alpha_{r r}$ and $\alpha_{\phi \phi}$, the symmetric/antisymmetric behaviour of the off-diagonal components are quite robust. The coefficients $\alpha_{r \phi}$ and $\gamma_{\theta}$ are anti-symmetric about the mid-plane at all radii. Rest of the off-diagonal components preserve symmetry about mid-plane which we discuss in section 4.2.1.

Till now, in this sub-section, we discussed how the spatial symmetry of the dynamo coefficients prevails at all radii. However, the most interesting result of the our SVD analysis in this subsection is that many of the calculated dynamo coefficients roughly follow a power-law of $\propto r^{-1.5}$. To guide reader, we draw a yellow solid line following a power-law $\propto r^{-1.5}$ in each panel of Fig. 3. In our simulations of the RIAF, we have $\Omega \sim r^{-1.7}$ and it is thus interesting that dynamo coefficients can have the similar radial dependence. Moreover, the coefficient crucial for the generation of poloidal field from toroidal one, $\alpha_{\phi \phi}$, is also negative in the $\mathrm{NH}$ and tends to change sign again further north. It would be important to understand these interesting features of $\alpha_{\phi \phi}$ from more basic theory in the future.

\subsubsection{Variation in the poloidal plane- a composite picture}

In the previous sub-sections, in order to study spatial dependence, we determined the meridional and radial variations of dynamo coefficients at the fixed radii and latitudes respectively. Fig. 4 shows a composite picture of spatial variation of dynamo coefficients in the $(r, \theta)$ plane. Panels at the top, middle and bottom show the spatial distribution of diagonal, symmetric off-diagonal and antisymmetric off-diagonal components of a tensor respectively. We put a black mask wherever the the error on the coefficients calculated by the SVD method exceeds its absolute value. It is quite clear in Fig. 4 that SVD works quite well in extracting dynamo coefficients at high latitudes where large-scale dynamo dominates. At low latitudes, the fluctuation dynamo dominates the evolution, the mean fields and EMFs are small, and dynamo coefficients associated with the large-scale dynamo are vanishingly small.

Regarding the distribution of dynamo coefficients in the poloidal plane, in a nutshell - apart from $\alpha_{\theta \theta}$, all other calculated dynamo coefficients show a coherent structure. While $\alpha_{r r}, \alpha_{\phi \phi}, \alpha_{r \phi}$ and $\gamma_{\theta}$ show a coherent antisymmetric behaviour about the midplane, $\alpha_{r \theta}, \alpha_{r \phi}, \alpha_{\theta \phi}, \gamma_{r}$ and $\gamma_{\phi}$ are coherently symmetric about the $\theta_{l}=0$ plane. Here, it must be mentioned that SVD treats each point in poloidal $(r, \theta)$ plane independently. Therefore, the symmetry/antisymmetry of dynamo coefficients about the mid-plane is not an artefact of the SVD method.

\subsection{Reliability check of the SVD method}

The SVD method provides an estimate of error on the determination of the coefficients $a_{i j}$. It is evident from Fig. 4 that at high latitudes, where mean fields, EMFs are stronger and dynamo coefficients are of non-zero values, variances are small. However, where mean fields and EMFs are vanishingly small (at low latitudes), errors are large (implying that a mean field description is invalid). In this subsection, we show some additional diagnostics to investigate the accuracy of the parameterization as recovered by the SVD method.

\subsubsection{Reconstruction of the EMF}

As a primary diagnostic, we reconstruct components of EMF using the extracted dynamo coefficients and mean magnetic fields (equation 7). Fig. 5 shows the comparison between the spatio-temporal 

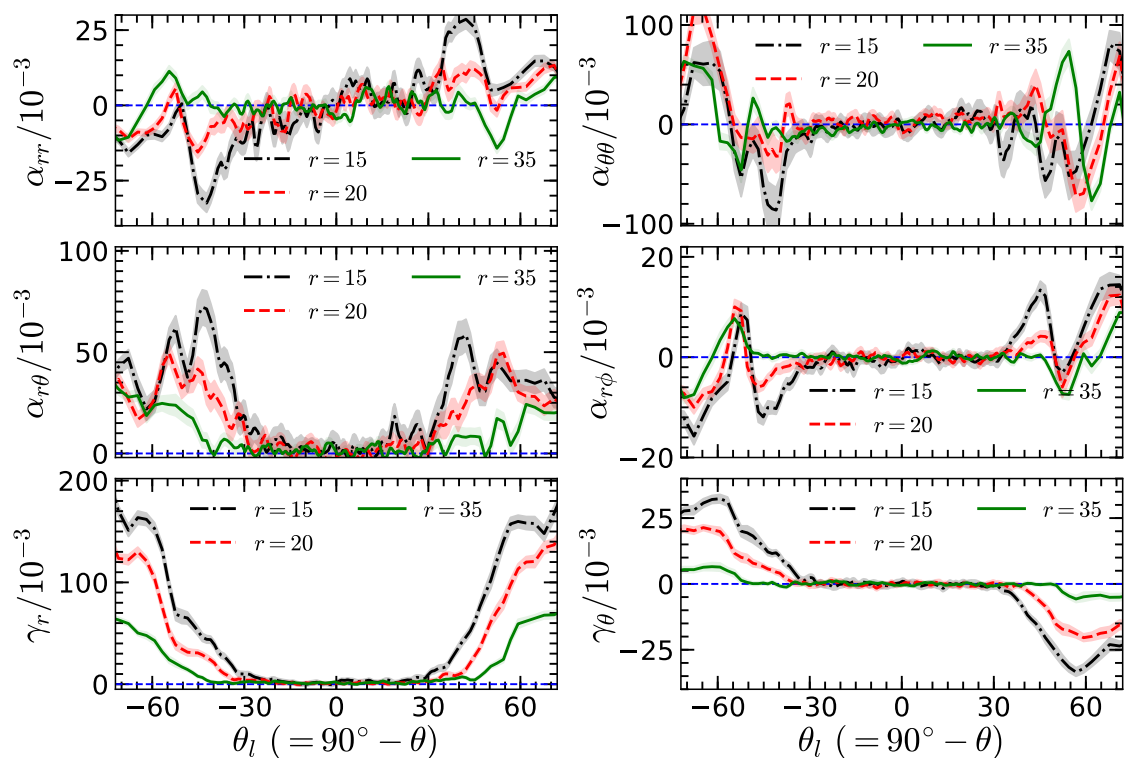

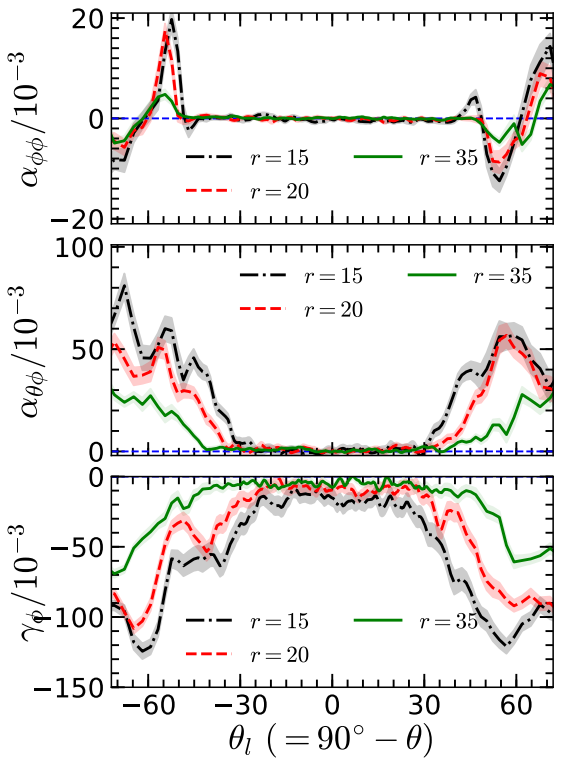

Figure 2. Meridional variation of dynamo coefficients at different radii $r=15,20,35$. Coloured region shows the corresponding $1 \sigma$ region of significance. Blue dashed horizontal line denotes zero of dynamo coefficients.
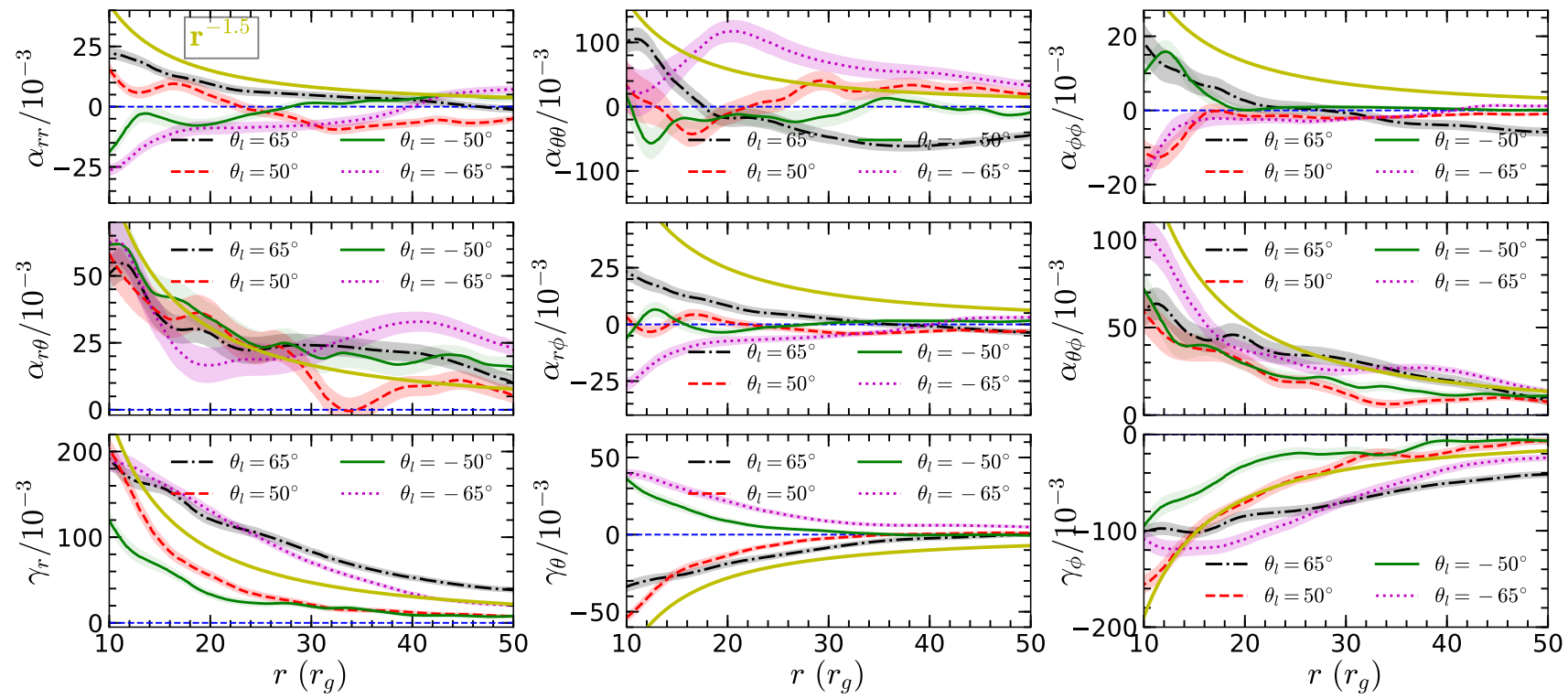

Figure 3. Variation of dynamo coefficients with radius at four different latitudes $\theta_{l}=-65^{\circ},-50^{\circ}, 50^{\circ}, 65^{\circ}$ in $\mathrm{NH}$ and $\mathrm{SH}$. Corresponding $1 \sigma$ region of significance is shown by the coloured error band. Blue dashed horizontal line denotes zero of dynamo coefficients. A power law profile of $\propto r^{-1.5}$ is shown by the yellow solid line.

variations of EMF (equation 5) obtained directly from the simulation (left panels) and reconstructed EMF $\overline{\mathcal{E}}^{M}$ (right panels) at a radius $r=20$. Top, middle and bottom panels of Fig. 5 show the butterfly diagrams for radial $\left(\overline{\mathcal{E}}_{r}\right)$, meridional $\left(\overline{\mathcal{E}}_{\theta}\right)$ and toroidal $\left(\overline{\mathcal{E}}_{\phi}\right)$ components of EMF respectively. It is evident that for $\overline{\mathcal{E}}_{r}$ and $\overline{\mathcal{E}}_{\phi}$ the match between the directly obtained EMF and reconstructed EMF is quite good at high latitudes, where the large-scale dynamo dominates. As expected, at low latitudes, the the reconstructed EMFs do not agree with the EMFs directly obtained from simulation because at low latitudes large scale dynamo is suppressed and mean fields, EMFs are vanishingly small. For $\overline{\mathcal{E}}_{\theta}$, agreement between the directly obtained and reconstructed EMFs is not as good as it is for $\overline{\mathcal{E}}_{r}$ and $\overline{\mathcal{E}}_{\phi}$. This poorer match is again because of the smallness of $\overline{\mathcal{E}}_{\theta}$ and $\bar{B}_{\theta}$.

\subsubsection{Residuals of EMFs obtained from simulations and reconstructed one}

Fig. 5 gives a good visual impression of agreement between the EMFs obtained directly from simulations and reconstructed using the mean field closure. To quantify the match, we calculate the residual of the directly obtained (from simulations) and reconstructed 

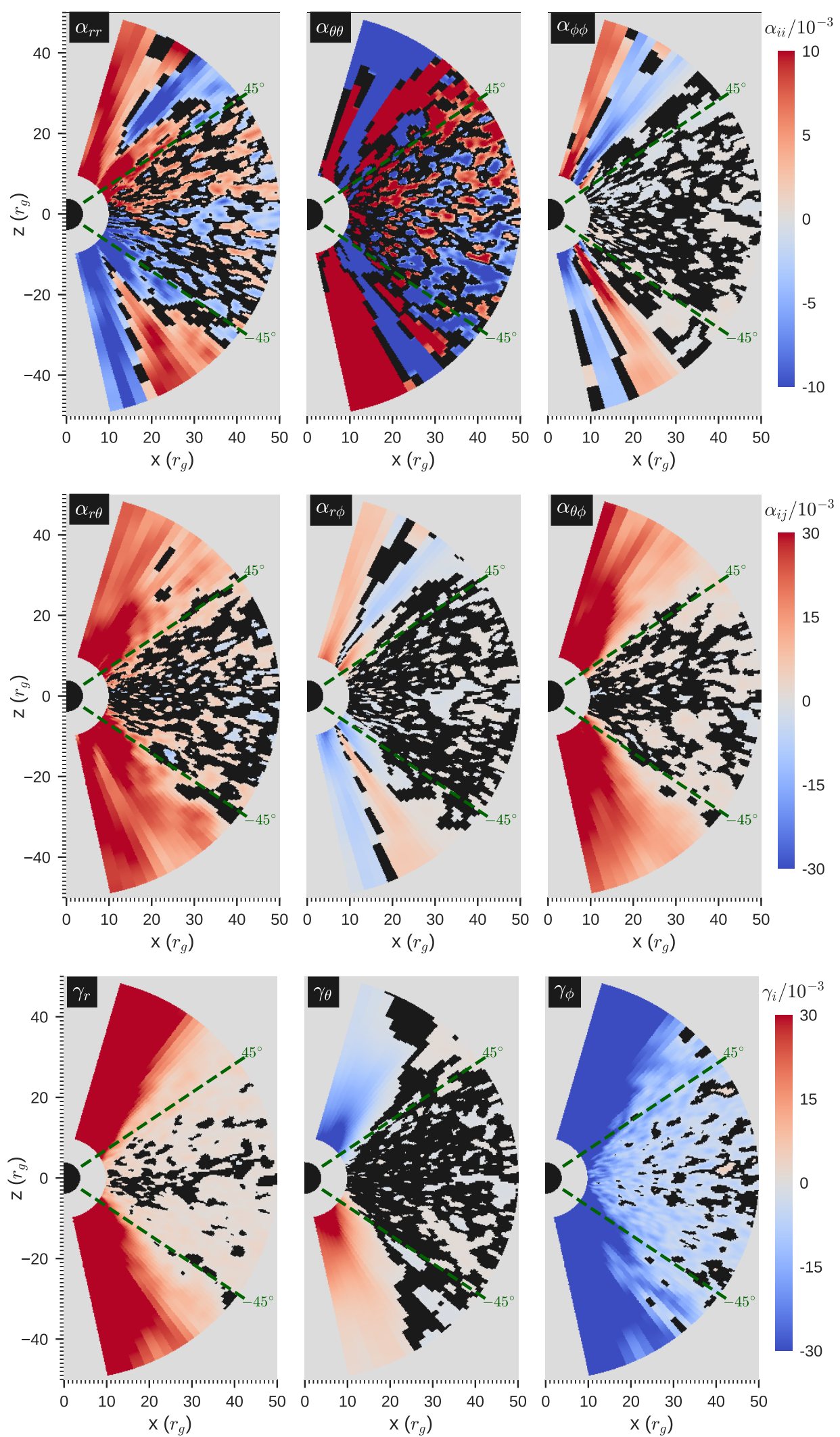

Figure 4. Poloidal distribution of different dynamo coefficients obtained by the SVD method using time series of the mean fields and EMFs in the quasi-steady state $\left(t_{\operatorname{sim}}[250,630]\right)$. The regions where the dynamo coefficients are smaller than one standard deviation, a black mask is applied. Green dashed lines on the $\mathrm{NH}$ and SH denote the latitudes $\theta_{l}=45^{\circ}$ and $\theta_{l}=-45^{\circ}$ respectively. Two of the diagonal $\left(\alpha_{r r}, \alpha_{\phi \phi}\right)$, and off-diagonal $\left(\alpha_{r \phi}\right.$ and $\left.\gamma_{\theta}\right)$ components are anti-symmetric about the mid-plane $\left(\theta_{l}=0^{\circ}\right)$. Rest of the off-diagonal coefficients $\left(\alpha_{r \theta}, \alpha_{\theta \phi}, \gamma_{r}\right.$ and $\left.\gamma_{\phi}\right)$ show symmetric behaviour about the mid-plane; $\alpha_{\theta \theta}$ is mildly symmetric about the mid-plane. Symmetry/antisymmetry of the dynamo coefficients about the mid-plane is not an artifact of the SVD method as the method treats each point in space independently. 

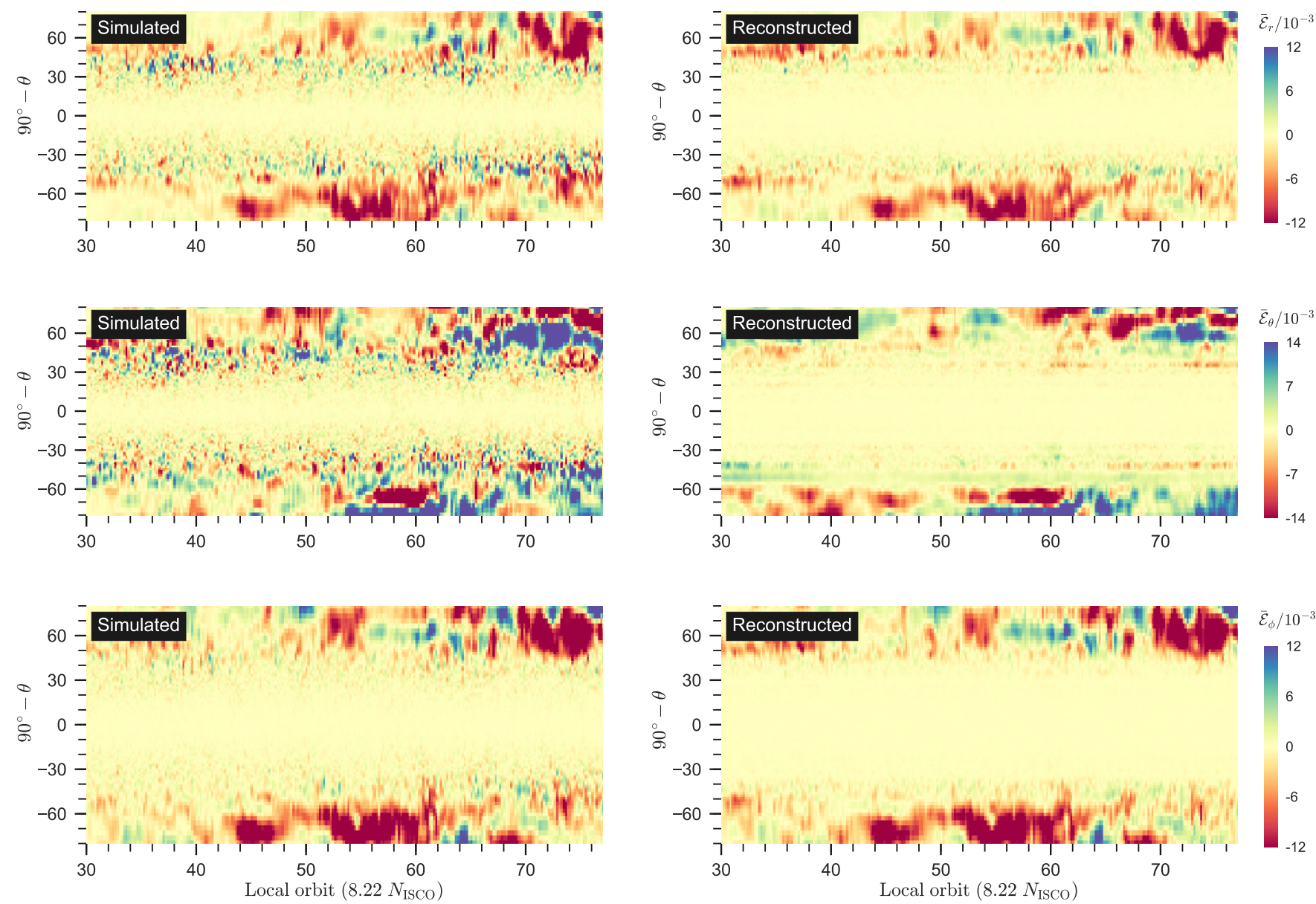

Figure 5. Spatio-temporal variation of radial (top panels), meridional (middle panels) and toroidal (bottom panels) components of simulated and reconstructed EMFs at a radial distance $R_{0}=20$. Radial and toroidal components show a good agreement between the simulated and reconstructed EMFs at high latitudes, where dynamo coefficients are of non-zero values. At low latitudes, the match is poor because at low latitudes large scale dynamo is suppressed and mean fields, EMFs are vanishingly small. The match between the simulated and reconstructed EMFs in the meridional component is not as good as for the other two components of EMFs.

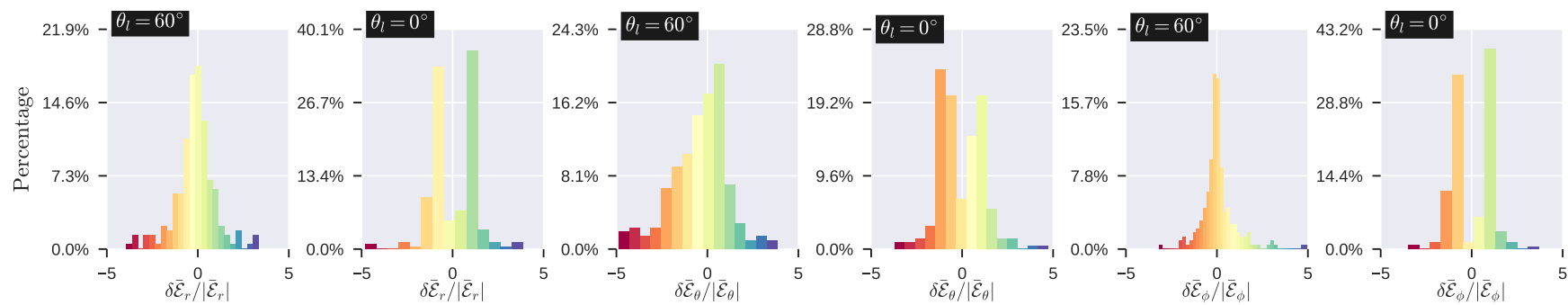

Figure 6. Histogram of the residuals of the simulated and reconstructed EMFs at two different latitudes $\theta_{l}=65^{\circ}$ (where large-scale dynamo dominates) and $\theta_{l}=0^{\circ}$ (where fluctuation dynamo operates) at $r=20$. Residual is divided by the magnitude of the simulated EMF. Note that the residuals are close to zero at high latitudes.

EMFs as follows

$$
\delta \overline{\mathcal{E}}_{i}(r, \theta, t)=\overline{\mathcal{E}}_{i}(r, \theta, t)-\overline{\mathcal{E}}_{i}^{M}(r, \theta, t) .
$$

Fig. 6 shows the histogram of residuals for each components of EMFs at a fixed radius $r=20$ and at two different latitudes- $\theta_{l}=$ $60^{\circ}$ and $\theta_{l}=0^{\circ}$. The residuals are normalised by the respective components of EMFs $\overline{\mathcal{E}}_{i}(r, \theta, t)$. It is easily observed that at high- latitudes, where the agreement is seen to be good in Fig. 5, the histogram shows a peak about zero. At low latitudes (mid-plane), where there is a large mis-match between $\overline{\mathcal{E}}_{i}$ and $\overline{\mathcal{E}}_{i}^{M}$ as seen in Fig. 5, there are few instances when the residuals are zero. It is also evident from the peak widths of the histograms that the match between $\overline{\mathcal{E}}_{i}$ and $\overline{\mathcal{E}}_{i}^{M}$ is best for $\phi$-component of EMF. 

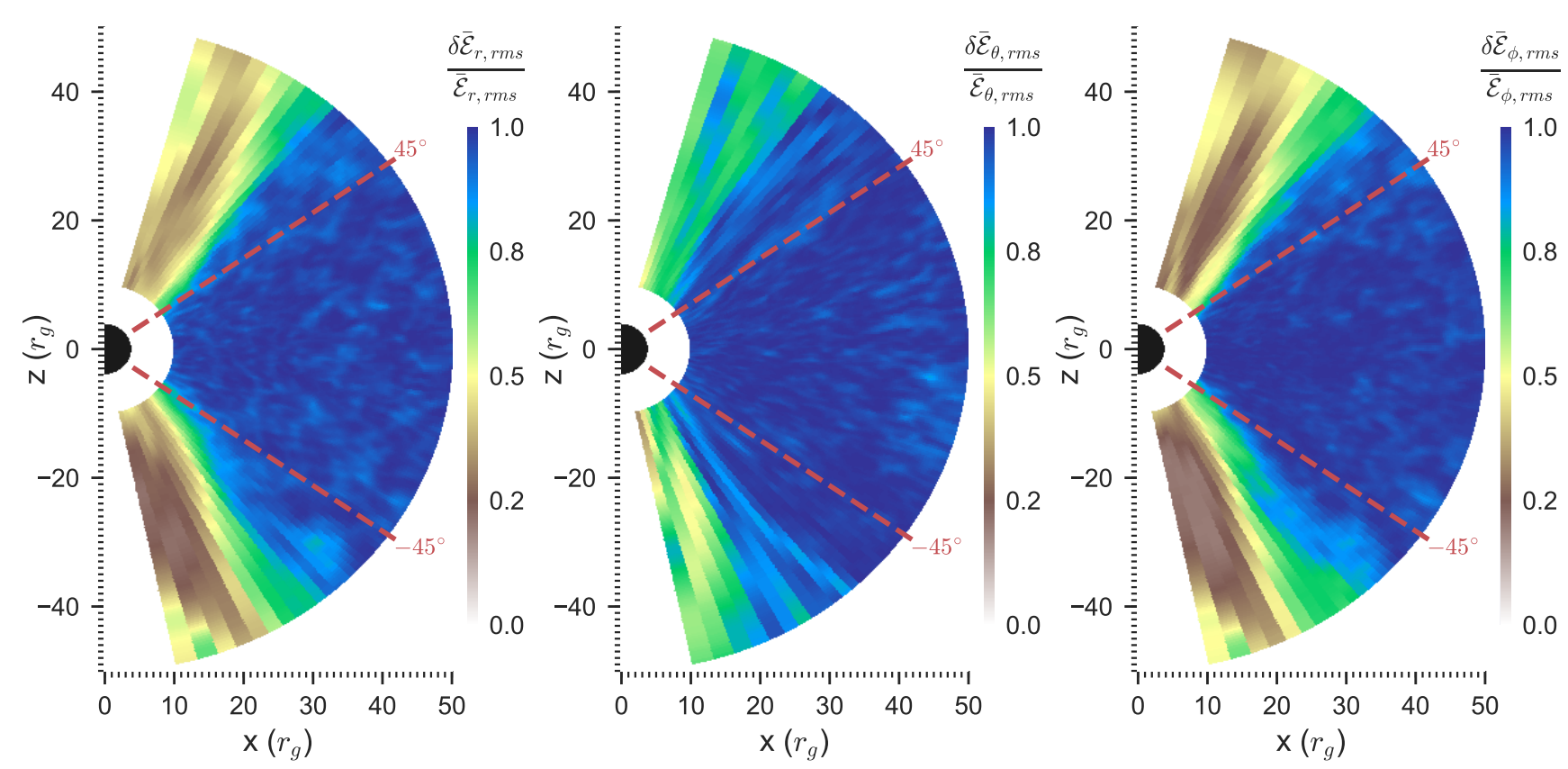

Figure 7. Distribution of rms values of the residuals between the reconstructed and simulated EMFs in the poloidal $(r, \theta)$ plane. Red dashed lines on northern and southern hemispheres denote the latitudes $\left(\theta_{l}=90^{\circ}-\theta\right) 45^{\circ}$ and $-45^{\circ}$ respectively. The rms values of residuals are normalised by the rms values of the corresponding component of the EMF. Residuals are smaller for both radial and toroidal components beyond a scale-height, but comparatively larger for the meridional component.

In Fig. 6, we investigate the agreement between simulated (directly obtained from the simulation) and reconstructed EMFs at different times at particular point in space. To see the how well the match is in a time-averaged sense over the whole poloidal plane, we calculate at the rms values of the residual EMFs. Fig. 7 shows the poloidal distribution of residuals $\delta \overline{\mathcal{E}}_{i, r m s}$. The residuals are normalised by the rms values of the EMFs $\overline{\mathcal{E}}_{i, r m s}$. Fig. 7 essentially echoes the key results shown in Fig. 5 and 6. We see that normalised residuals are minimum at high latitudes and maximum around the mid-plane. Thus SVD provides a good match between the reconstructed and simulated EMFs at high latitudes where a large scale dynamo dominates. This also indicates that the $b_{i j k}$ tensor does not make a statistically significant contribution to the EMF as we had stated earlier. The match between the two EMFs is poor at low latitudes as expected, where a turbulent small-scale dynamo operates.

\section{DISCUSSION}

\subsection{Comparison with previous local and global studies}

All the previous global studies (Arlt \& Rüdiger 2001; Flock et al. 2012; Hogg \& Reynolds 2018) consider a simpler closure

$$
\overline{\mathcal{E}}_{\phi} \approx \alpha_{\phi \phi} \bar{B}_{\phi}
$$

to characterise the dynamo coefficients in accretion flows. In this paper, we use a more general closure to calculate the diagonal as well as the off-diagonal dynamo coefficients in a global simulation of accretion flow around the black hole. Determination of dynamo coefficients throughout the poloidal plane $(r, \theta)$ gives us the opportunity to compare our findings with the previous local and global studies.

We use the spherical polar coordinates $(r, \theta, \phi)$ in our global simulations. Previous shearing box studies (Brandenburg 2008; Gressel 2010; Gressel \& Pessah 2015) characterising dynamo coefficients use the TF method. For comparison, we can make a mapping from spherical polar to Cartesian coordinates for the three dynamo coefficients; $\alpha_{x x} \rightarrow \alpha_{r r}, \alpha_{y y} \rightarrow \alpha_{\phi \phi}$ and $\gamma_{z} \rightarrow \gamma_{\theta}$. The trend in the meridional variation of these three coefficients agrees well between local shearing box simulations (see Fig. 5 of Gressel (2010)) and our global study (see Fig. 2). For example, like $\alpha_{y y}$, its spherical counterpart $\alpha_{\phi \phi}$ is negative / positive close the mid-plane and tends to be positive/ negative at higher latitudes in the northern hemisphere $(\mathrm{NH})$ /southern hemisphere (SH). However, to be confident about prevalence of positive sign at high latitudes, the calculation needs to be done for a geometrically thin disc $(H / R \ll 1)$, where many scale-heights are available.

There is a long standing disagreement between local and global simulations regarding the sign of $\alpha_{\phi \phi}$, calculated using the simple closure (equation 23). Local studies (Brandenburg et al. 1995; Brandenburg \& Donner 1997; Davis et al. 2010) found a negative $\alpha_{\phi \phi}$ in NH. Global studies (Arlt \& Rüdiger 2001; Flock et al. 2012) found an opposite trend, i.e a positive $\alpha_{\phi \phi}$ in NH. However, Hogg $\&$ Reynolds (2018) obtained an $\alpha_{\phi \phi}$ of negative sign at $r=15$ and between $+H$ to $+2 H$ (i.e in $\mathrm{NH}$ ) using the same simple closure (equation 23). The reason behind the agreement/disagreement among the different studies especially among the recent global studies (Flock et al. 2012; Hogg \& Reynolds 2018 and the current work) is not very clear. All three works use the same code (PLUTO) and similar combination of algorithms recommended by Flock et al. (2010).

\subsection{Opportunity to build an effective mean field model}

To attain saturation of the MRI and for dynamo action, an expensive 3D simulation has to be performed for a sufficiently long time. 


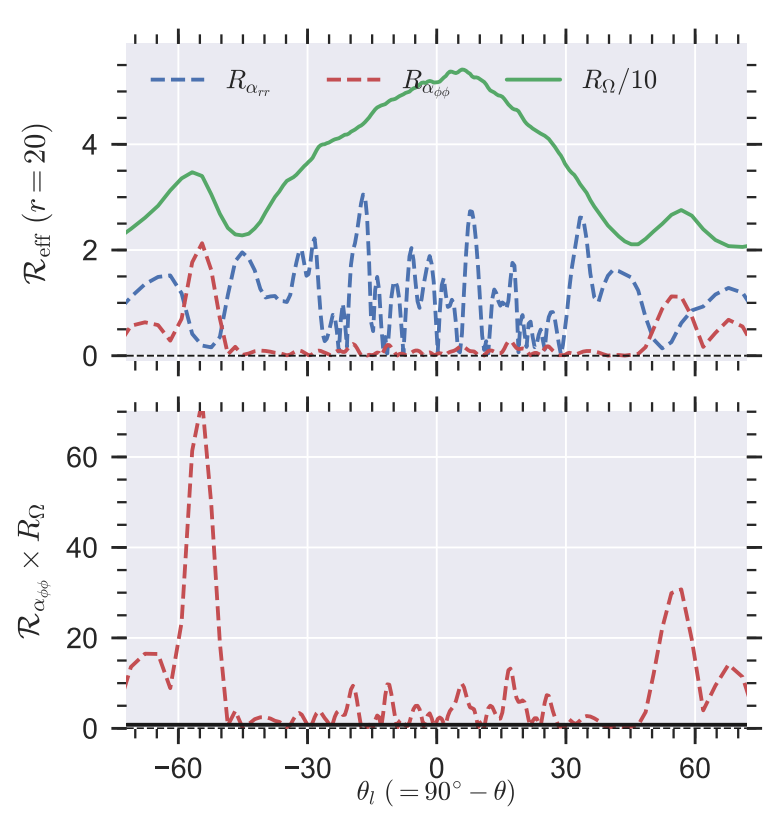

Figure 8. Top panel: Meridional variation of the control parameters related to the measurement of the strength of $\alpha$-effect (equations 24) and shear (25) at $r=20$. Bottom panel: Meridional variation of the dynamo number $D=R_{\alpha_{\phi \phi}} R_{\Omega}$ at $r=20$.

Determination of the spatial profiles of dynamo coefficients gives an opportunity to build an effective mean field model for MRI driven accretion flow, which can be run in 2D with sustained turbulence. In particular, an effective mean field model can be useful in simulating the expensive geometrically thin $\operatorname{disc}(H / R \ll 1)$ with a long dynamical range.

There have been previous attempts to build an effective mean field models for MRI driven accretion flow (Bucciantini \& Del Zanna 2013; Stepanovs et al. 2014; Sądowski et al. 2015; Fendt \& Gaßmann 2018; Tomei et al. 2020). However, all those models consider either an $\alpha$-effect or both $\alpha$-effect and turbulent diffusivity using some simple closure. Consideration of the full set of dynamo coefficients in the mean field model is expected to provide a more realistic result.

\subsection{Characterisation of the dynamo}

Dynamo action in the differentially rotating accretion disc is traditionally categorised as an $\alpha-\Omega$ dynamo (Brandenburg et al. 1995; Gressel 2010). Here, $\Omega$ effect refers to the generation of toroidal component of magnetic field from the poloidal components by differential rotation. Poloidal field can be regenerated from the toroidal field by an $\alpha$-effect. The coefficient $\alpha_{\phi \phi}$ is responsible for the conversion of toroidal fields into poloidal fields. The coefficients $\alpha_{r r}$ and $\alpha_{\theta \theta}$ are related to generation of toroidal fields by the $\alpha$-effect. However, the regeneration of the toroidal field from poloidal field is dominated by strong shear, or the $\Omega$-effect in accretion discs. Therefore these dynamos are referred to as $\alpha-\Omega$ dynamos.

We quantify the strength of such a dynamo by using the meanfield induction equation to identify different dimensionless control parameters. Firstly, the parameter $R_{\alpha_{i j}}=\alpha_{i j} H / \eta_{T}$ measures the importance of the $\alpha$-effect to amplify the field against turbulent diffusion $\eta_{T}$. The importance of the shear is governed by the parameter $R_{\Omega}=S H^{2} / \eta_{T}$. The product $D=R_{\alpha_{\phi \phi}} R_{\Omega}$ called the dynamo number, measures the combined amplification strength of both the $\alpha$ and $\Omega$ effects in an $\alpha-\Omega$ dynamo. It should exceed a critical value $D_{\text {crit }}$ for amplification of the field, which for thin discs is $\sim 10$ (Brandenburg \& Subramanian 2005). Of course the value of $D_{\text {crit }}$ for the RIAF is not known apriori. The value of shear $S=1.5 \Omega$ and scale-height $H=c_{s} / \Omega$, are known from the simulation data, while $\alpha_{i j}$ can be obtained from the SVD analysis. Turbulent diffusion is approximated as $\eta_{T}=v_{r m s}^{\prime} / 3 k_{\mathrm{MRI}}$, where we are motivated from the TF result on forced turbulence (Sur et al. 2008), with $k_{M R I}=\Omega / v_{A}$, the wavenumber of the fastest growing mode of MRI. This leads to an estimate,

$$
\begin{aligned}
R_{\alpha_{i j}} & =\frac{\alpha_{i j} H}{\eta_{T}}=3 \sqrt{\frac{\beta}{2}} \frac{\alpha_{i j}}{v_{r m s}^{\prime}}, \\
R_{\Omega} & =\frac{S H^{2}}{\eta_{T}}=\frac{9}{4} \beta \frac{v_{A}}{v_{r m s}^{\prime}},
\end{aligned}
$$

where $\beta=2 P_{\text {gas }} / B^{2}$.

These numbers are generally studied in the kinematic stage; but here we calculate them here in saturation. Top and bottom panels of Fig. 8 show the meridional variation of the control parameters $\left(R_{\alpha_{\phi \phi}}, R_{\alpha_{r r}}\right.$ and $\left.R_{\Omega}\right)$ and dynamo number $D=R_{\alpha_{\phi \phi}} R_{\Omega}$ with latitude respectively. Here, we consider only $\alpha_{r r}$ and $\alpha_{\phi \phi}$, related to the dominant components of magnetic field $B_{r}$ and $B_{\phi}$ respectively. It is evident from Fig. 8 that at all latitudes $R_{\Omega}>R_{\alpha_{i j}}$ so that dynamo generation is controlled by the dynamo number $D=R_{\alpha_{\phi \phi}} R_{\Omega}$. This is of course a local value of $D$ at some $(r, \theta)$ and to infer whether the dynamo is super critical, one needs to solve the mean-field induction equation with these $\alpha_{i j}$ and shear profiles. But it is interesting to note from the the meridional profile of the local value of $D$, that it exceeds about 10 where the mean field is prominent. More work solving the mean-field dynamo equation is needed to put this conclusion on a firmer footing. We expect the dynamo action to be local because both the kinetic and current helicities become non-vanishing at high latitudes (Fig. 26 in Dhang \& Sharma (2019)) where a large-scale dynamo operates and dynamo coefficients have non-zero values.

\subsection{Turbulent pumping, radially outward transport of the mean field}

Studies (Lubow et al. 1994; Guilet \& Ogilvie 2013) which consider the accretion of a large-scale poloidal field on to the central black hole consider turbulent diffusion as the only factor opposing inward advection of magnetic flux. Importance of the turbulent pumping in mean magnetic flux transport was previously unnoticed. In our study, we find that along with turbulent diffusion, turbulent pumping can also act against inward advection. Fig. 4 shows $\gamma_{\theta}$ that represents the transport of the mean magnetic field from turbulent region close to the mid-plane to the less turbulent coronal region. On the other hand, $\gamma_{r}$ shows that the mean field is transported radially outward. The direction of transport is down the gradient of turbulence intensity which we find decreases with increasing radius, as $v_{r m s}^{\prime} \sim r^{-1}$.

To see the net effect of these two coefficients, we construct a vector quantity $\gamma_{p}=\gamma_{r} \hat{r}+\gamma_{\theta} \hat{\theta}$. Fig 9 shows the distribution of $\gamma_{p}$ in the poloidal plane. Colour describes the magnitude and arrow, the direction. A black mask is applied wherever the standard deviation is larger than the magnitude of $\gamma_{p}$. Fig. 9 clearly shows that the large scale field is transported radially outward due to turbulent diamagnetism at high latitudes. Fig. 10 shows the comparison of 


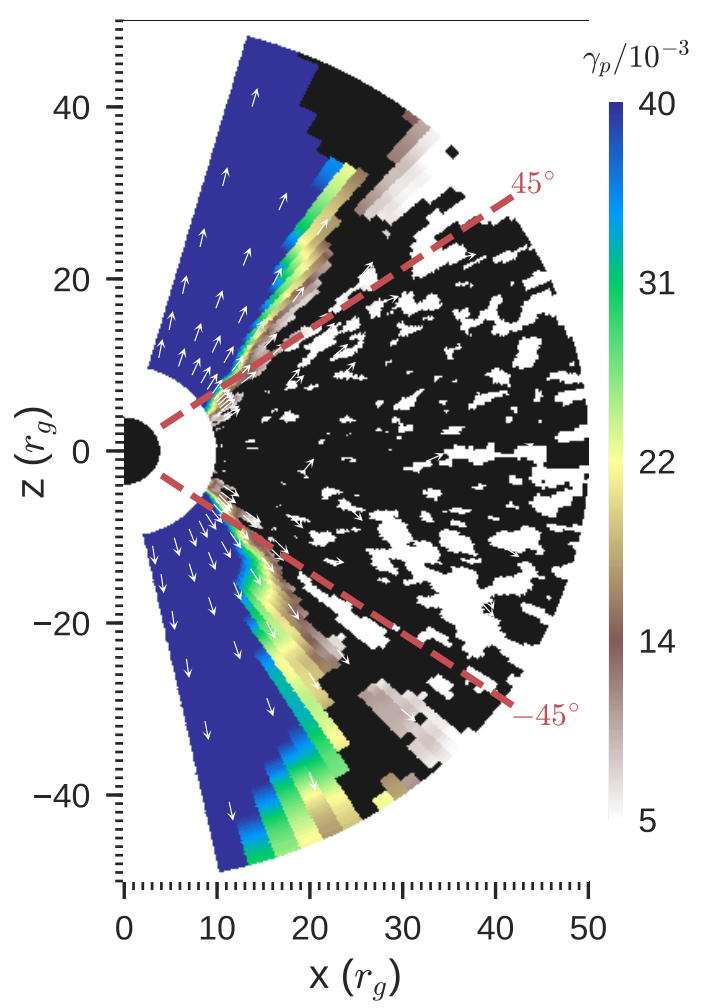

Figure 9. Distribution of $\gamma_{\boldsymbol{p}}=\gamma_{r} \hat{r}+\gamma_{\theta} \hat{\theta}$ in the poloidal plane. Colour and arrows denote the magnitude and direction of $\gamma_{p}$ respectively. A black mask is applied in the regions where the ratio of ratio of standard deviation to magnitude of the quantity is greater than the unity.

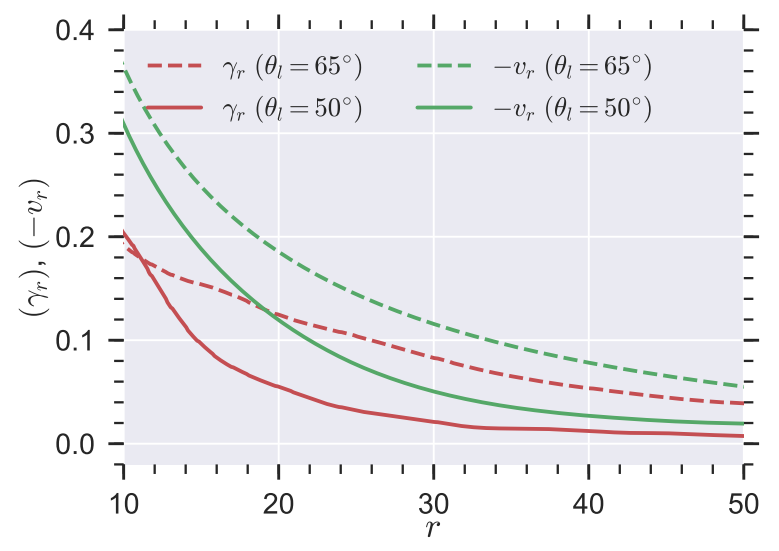

Figure 10. Comparison of radial turbulent pumping $\gamma_{r}(r)$ and radial advection $\bar{v}_{r}(r)$ at two different latitudes $\theta_{l}=50^{\circ}, 65^{\circ}$. Radially outward turbulent pumping is significant enough to oppose the inward advection of magnetic flux by the mean flow.

radial profiles of turbulent pumping $\gamma_{r}$ and radial advection $\bar{v}_{r}$ at two different latitudes $\theta_{l}=55^{\circ}, 65^{\circ}$. At all radii, $\gamma_{r}$ is comparable to $\bar{v}_{r}$, the difference decreases with an increasing radius. We expect the effect of turbulent pumping to be stronger in case of a geometrically thin disc $(H / R \ll 1)$ where the radial velocity (even in the coronal region) is smaller.

Recent net flux simulations of a geometrically thin Keplerian disc also show that magnetic flux does not accumulate efficiently near the centre due to the quasi-steady balance of advection and diffusion of magnetic fields (Zhu \& Stone 2018; Mishra et al. 2019). Our study suggests that this may be due to the presence of a strong turbulent pumping rather than simply turbulent diffusion. However, flux accumulation can be efficient if the RIAF is magnetically arrested (MAD; Bisnovatyi-Kogan \& Ruzmaikin 1974; Igumenshchev et al. 2003; Narayan et al. 2003; Tchekhovskoy et al. 2011; McKinney et al. 2012). In case of a MAD, the strong coherent large-scale magnetic field is dynamically important and the dynamo is completely suppressed (e.g. see Fig. 16 of McKinney et al. (2012)) and the large scale field is efficiently dragged in.

\subsection{Dynamo action and the jet connection}

Jets are observed in hard spectral state in BHBs. The basic requirements to produce the jet are a sufficiently strong, ordered poloidal magnetic field and an inner hot geometrically thick accretion flow (e.g. see Meier (2005)). However, numerical simulations show that there is no apparent correlation between the disc scale height and jet power (Fragile et al. 2012). This leads to the possibility that magnetic field geometry might be the deciding factor in determining the jet power, although, association of jets with low/hard state implies the presence of a RIAF close to the black hole (Fender et al. 1999). In section 5.4, we discussed the difficulty in transporting large-scale magnetic fields inward close to the black hole unless accretion occurs in the magnetically dominated regime.

In this section, we propose a scenario, in which a large scale mean field dynamo operates in the outer thin disc and seeds a MAD state in the inner RIAF. ${ }^{1}$ This magnetic arrangement is conducive to jet launching. Fig. 11 shows the accretion flow geometry in the truncated disc model (Esin et al. 1997; Done et al. 2007). According to this model, the outer standard disc is truncated to join with an inner RIAF over a transition radius $r_{t r} \approx 30-200 r_{g}$ (Nemmen et al. 2014). In the outer thin disc, dynamo action is strong, and magnetic fields flip with a dynamo period $T_{\text {dyn }} \approx 10-15 T_{\text {orb }} \propto r^{3 / 2}$ which is much longer than the timescale at ISCO where a jet is launched. Hence, innermost part of the outer standard disc can supply coherent large-scale $(\sim H)$ dynamo generated ordered magnetic fields to the inner RIAF leading to a magnetically dominated accretion flow and hence a favourable condition for jets.

It should be mentioned that the generation of a MAD depends on the size of the inner RIAF. If area occupied by the inner RIAF shrinks and inner disc moves inward, the polarity of the supplied field flips more quickly as $T_{\mathrm{dyn}} \propto r^{3 / 2}$. Therefore, time span over which mean fields of the same polarity is supplied to inner RIAF is shorter. The supply of opposite polarity fields enhances reconnection within the RIAF and the disc becomes less magnetised and can lead to the transient ballistic jets from the region close to the black hole (Dexter et al. 2014). However, few studies (e.g Stepanovs et al. 2014) associate episodic jets with the duty cycles of the dynamo in the thin disc itself. In the soft state, a thin disc extends till the ISCO. Efficient dynamo action in the thin disc close to the black hole leads to the frequent polarity reversal of the dynamo generated large scale magnetic fields. Hence, the jet is quenched due to the weakening of coherent large scale poloidal fields.

1 Although turbulent pumping gives outward transport of mean fields, the mean inflow speed dominates and causes inward advection of a large-scale poloidal field in the coronal region (Figure 10). 


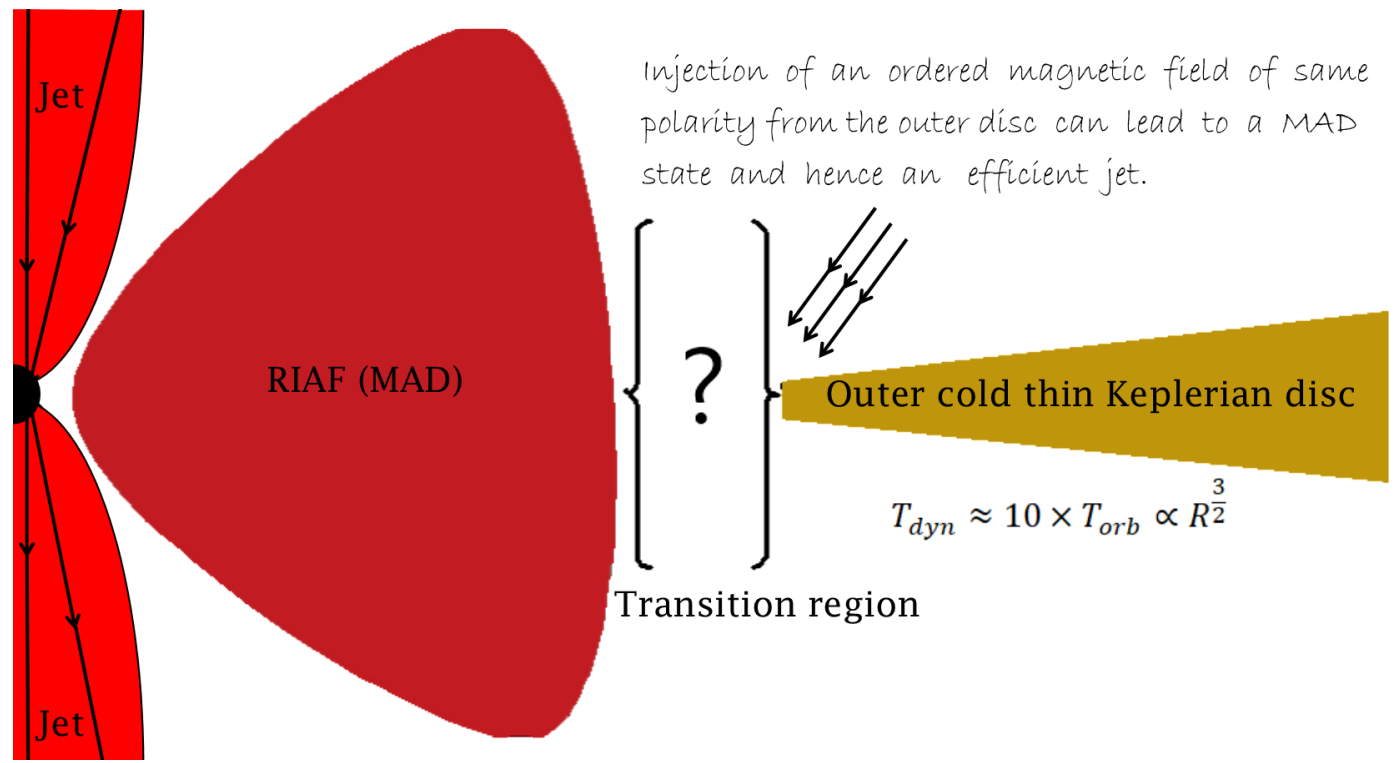

Figure 11. Cartoon diagram of the geometry of the accretion flow in the low/hard state of BHBs in the truncated disc model. The model requires a outer cold, optically thick, geometrically thin disc and an inner hot, optically thin, geometrically thick flow (RIAF). While jets are present in the low/hard state, they are absent in the high/soft state. Dynamo generated large-scale $(\sim H \propto r)$ field produced at the inner edge of the outer thin disc can be supplied to the inner RIAF to establish a magnetically dominated RIAF that aids formation of jets.

\section{SUMMARY}

In this paper, we have analysed the MRI driven dynamo in a weakly magnetised radiatively inefficient accretion flow (RIAF). We use the mean field dynamo formalism to understand mechanism for the generation of large-scale magnetic fields in the RIAF (model M-2P discussed in Dhang \& Sharma (2019)). The key results of our work are summarised below.

- We have recovered the dynamo coefficients for the MRI driven large-scale dynamo in the RIAF using the SVD method. Our study is the first one to calculate the distribution of dynamo coefficients in the poloidal plane $(r, \theta)$. Out of the calculated coefficients, four coefficients $\alpha_{r r}, \alpha_{\phi \phi}, \alpha_{r \phi}$ and $\gamma_{\theta}$ are anti-symmetric about the mid-plane $\left(\theta_{l}=90^{\circ}-\theta=0^{\circ}\right)$. Rest of the coefficients $\left(\alpha_{r \theta}, \alpha_{\theta \phi}\right.$, $\gamma_{r}$ and $\gamma_{\phi}, \alpha_{\theta \theta}$ ) show symmetric behaviour about the mid-plane. Many of the the calculated coefficients roughly scale as a power law $\propto r^{-1.5}$, similar to the angular velocity $\Omega \propto r^{-1.7}$.

- We find that meridional variations of $\alpha_{\phi \phi}$, responsible for toroidal to poloidal field conversion and considered to be the most important dynamo coefficient, is very similar to that found in shearing box simulations using the 'test field method'. The dynamo coefficient $\alpha_{\phi \phi}$ is negative at low latitudes and tends to be positive at higher latitudes.

- We estimate the relative importance of the $\alpha$-effect and shear. We conclude that although $\alpha_{r r}$ is quite large, the MRI driven largescale dynamo is essentially of the $\alpha-\Omega$ type.

- The information of dynamo coefficients will allow us to carry out less expensive axisymmetric global accretion disc simulations with sustained turbulence. Simulating such an effective mean field model is especially attractive in context of geometrically thin disc with a large dynamical range.

- We find a strong turbulent pumping, which transports large scale magnetic fields radially outward. This effect along with the turbulent diffusion makes it difficult for the large-scale magnetic fields, an important factor to produce jets, to be advected inward by the mean flow.
- We propose a mechanism to explain the presence of jets in the low/hard state of a BHB considering a truncated disc model. We outline a scenario where a dynamo generated large-scale field produced at the inner edge of the outer thin disc can be supplied to the inner RIAF to establish a magnetically dominated RIAF (MAD) conducive to jet formation.

\section{ACKNOWLEDGEMENTS}

PD would like to thank Xue-Ning Bai, Paul Charbonneau and Bhupendra Mishra for useful discussions. We thank Oliver Gressel for detailed comments on the manuscript. We thank the reviewer for the useful comments. PD would also like to thank IUCAA for the hospitality extended to him during his multiple visits to the institute. PS acknowledges a Swarnajayanti Fellowship from the Department of Science and Technology (DST/SJF/PSA- 03/2016-17). The simulations analysed in this paper were carried out on Cray XC40-SahasraT cluster at Supercomputing Education and Research Centre (SERC), IISc.

\section{REFERENCES}

Arlt R., Rüdiger G., 2001, A\&A, 374, 1035

Bai X.-N., Stone J. M., 2013, ApJ, 769, 76

Balbus S. A., Hawley J. F., 1991, ApJ, 376, 214

Bendre A. B., Subramanian K., Elstner D., Gressel O., 2019, MNRAS

Bhat P., Ebrahimi F., Blackman E. G., 2016, MNRAS, 462, 818

Bisnovatyi-Kogan G. S., Ruzmaikin A. A., 1974, Ap\&SS, 28, 45

Bisnovatyi-Kogan G. S., Ruzmaikin A. A., 1976, Ap\&SS, 42, 401

Blackman E. G., Nauman F., 2015, Journal of Plasma Physics, 81, 395810505

Blandford R. D., Begelman M. C., 1999, MNRAS, 303, L1

Blandford R. D., Payne D. G., 1982, MNRAS, 199, 883

Blandford R. D., Znajek R. L., 1977, MNRAS, 179, 433

Brandenburg A., 2008, Astronomische Nachrichten, 329, 725

Brandenburg A., 2009, Space Sci. Rev., 144, 87 
Brandenburg A., Donner K. J., 1997, MNRAS, 288, L29

Brandenburg A., Subramanian K., 2005, Phys. Rep., 417, 1

Brandenburg A., Nordlund A., Stein R. F., Torkelsson U., 1995, ApJ, 446, 741

Bucciantini N., Del Zanna L., 2013, MNRAS, 428, 71

Cao X., 2018, MNRAS, 473, 4268

Chandrasekhar S., 1960, Proceedings of the National Academy of Science, 46,253

Davis S. W., Stone J. M., Pessah M. E., 2010, ApJ, 713, 52

Dexter J., McKinney J. C., Markoff S., Tchekhovskoy A., 2014, MNRAS, 440, 2185

Dhang P., Sharma P., 2019, MNRAS, 482, 848

Done C., Gierliński M., Kubota A., 2007, A\&ARv, 15, 1

Esin A. A., McClintock J. E., Narayan R., 1997, ApJ, 489, 865

Fender R., et al., 1999, ApJ, 519, L165

Fendt C., Gaßmann D., 2018, ApJ, 855, 130

Flock M., Dzyurkevich N., Klahr H., Mignone A., 2010, A\&A, 516, A26

Flock M., Dzyurkevich N., Klahr H., Turner N., Henning T., 2012, ApJ, 744, 144

Fragile P. C., Wilson J., Rodriguez M., 2012, MNRAS, 424, 524

Gressel O., 2010, MNRAS, 405, 41

Gressel O., Pessah M. E., 2015, ApJ, 810, 59

Guilet J., Ogilvie G. I., 2012, MNRAS, 424, 2097

Guilet J., Ogilvie G. I., 2013, MNRAS, 430, 822

Hawley J. F., Gammie C. F., Balbus S. A., 1996, ApJ, 464, 690

Hawley J. F., Richers S. A., Guan X., Krolik J. H., 2013, ApJ, 772, 102

Hogg J. D., Reynolds C. S., 2018, ApJ, 861, 24

Igumenshchev I. V., Narayan R., Abramowicz M. A., 2003, ApJ, 592, 1042

Jiang Y.-F., Stone J. M., Davis S. W., 2014, ApJ, 784, 169

Johansen A., Levin Y., 2008, A\&A, 490, 501

Krause F., Rädler K. H., 1980, Mean-Field Magnetohydrodynamics and Dynamo Theory. Pergamon Press (also Akademie-Verlag: Berlin), Oxford

Liska M. T. P., Tchekhovskoy A., Quataert E., 2018, arXiv e-prints

Lubow S. H., Papaloizou J. C. B., Pringle J. E., 1994, MNRAS, 267, 235

Mandel J., 1982, The American Statistician, 36, 15

McKinney J. C., Tchekhovskoy A., Blandford R. D., 2012, MNRAS, 423, 3083

Meier D. L., 2005, Ap\&SS, 300, 55

Mignone A., Bodo G., Massaglia S., Matsakos T., Tesileanu O., Zanni C., Ferrari A., 2007, ApJS, 170, 228

Mishra B., Begelman M. C., Armitage P. J., Simon J. B., 2019, arXiv e-prints

Moffatt H. K., 1978, Magnetic Field Generation in Electrically Conducting Fluids. Cambridge Univ. Press, Cambridge

Narayan R., Yi I., 1994, ApJ, 428, L13

Narayan R., Igumenshchev I. V., Abramowicz M. A., 2000, ApJ, 539, 798

Narayan R., Igumenshchev I. V., Abramowicz M. A., 2003, PASJ, 55, L69

Nauman F., Blackman E. G., 2015, MNRAS, 446, 2102

Nemmen R. S., Storchi-Bergmann T., Eracleous M., 2014, MNRAS, 438, 2804

Paczyńsky B., Wiita P. J., 1980, A\&A, 88, 23

Papaloizou J. C. B., Pringle J. E., 1984, MNRAS, 208, 721

Parkin E. R., Bicknell G. V., 2013, MNRAS, 435, 2281

Penna R. F., Narayan R., Sądowski A., 2013, MNRAS, 436, 3741

Press W. H., Teukolsky S. A., Vetterling W. T., Flannery B. P., 1992, Numerical recipes in $\mathrm{C}$. The art of scientific computing

Quataert E., Gruzinov A., 2000, ApJ, 539, 809

Racine É., Charbonneau P., Ghizaru M., Bouchat A., Smolarkiewicz P. K., 2011, ApJ, 735, 46

Rädler K. H., 1969, Veroeffentlichungen der Geod. Geophys, 13, 131

Sądowski A., Narayan R., Tchekhovskoy A., Abarca D., Zhu Y., McKinney J. C., 2015, MNRAS, 447, 49

Schrinner M., RÃd'dler K.-H., Schmitt D., Rheinhardt M., Christensen U. R., 2007, Geophysical \& Astrophysical Fluid Dynamics, 101, 81

Simard C., Charbonneau P., Dubé C., 2016, Advances in Space Research, 58,1522

Stepanovs D., Fendt C., Sheikhnezami S., 2014, ApJ, 796, 29

Sur S., Brandenburg A., Subramanian K., 2008, MNRAS, 385, L15

Suzuki T. K., Inutsuka S.-i., 2014, ApJ, 784, 121
Tchekhovskoy A., Narayan R., McKinney J. C., 2011, MNRAS, 418, L79 Tomei N., Del Zanna L., Bugli M., Bucciantini N., 2020, MNRAS, 491, 2346

Velikhov E., 1959, Sov. Phys. JETP, 36, 995

Yuan F., Narayan R., 2014, ARA\&A, 52, 529

Yuan F., Bu D., Wu M., 2012, ApJ, 761, 130

Zhu Z., Stone J. M., 2018, ApJ, 857, 34

\section{APPENDIX A: COMPLETE SET OF DYNAMO COEFFICIENTS}

Without neglecting the contribution of $\mathbf{b}$ tensor in the expansion of equation $6, i^{\text {th }}$ component of EMF can be written as

$$
\overline{\mathcal{E}}_{i}=\tilde{a}_{i j} \bar{B}_{j}+\tilde{b}_{i j r} \frac{\partial \bar{B}_{j}}{\partial r}+\frac{\tilde{b}_{i j \theta}}{r} \frac{\partial \bar{B}_{j}}{\partial \theta},
$$

and the components of pseudo tensors $\tilde{a}_{i j}$ and $\tilde{b}_{i j}$ can then be used to express the following complete set of dynamo coefficients including the diffusive terms, that depend explicitly on the components of $\mathbf{b}$,

$$
\begin{aligned}
& \alpha_{r r}=\tilde{a}_{r r}-\frac{\tilde{b}_{r \theta \theta}}{r}, \\
& \alpha_{\theta \theta}=\tilde{a}_{\theta \theta}+\frac{\tilde{b}_{\theta r \theta}}{r}, \\
& \alpha_{\phi \phi}=\tilde{a}_{\phi \phi}, \\
& \alpha_{r \theta}=\alpha_{\theta r}=\frac{1}{2}\left(\tilde{a}_{r \theta}+\tilde{a}_{\theta r}+\frac{\tilde{b}_{r r \theta}}{r}-\frac{\tilde{b}_{\theta \theta \theta}}{r}\right), \\
& \alpha_{r \phi}=\alpha_{\phi r}=\frac{1}{2}\left(\tilde{a}_{r \phi}+\tilde{a}_{\phi r}-\frac{\tilde{b}_{\phi r r}}{r}\right) \text {, } \\
& \alpha_{\theta \phi}=\alpha_{\phi \theta}=\frac{1}{2}\left(\tilde{a}_{\theta \phi}+\tilde{a}_{\phi \theta}+\frac{\tilde{b}_{\phi r \theta}}{r}\right) \text {. } \\
& \gamma_{r}=\frac{1}{2}\left(\tilde{a}_{\theta \phi}-\tilde{a}_{\phi \theta}-\frac{\tilde{b}_{\phi r \theta}}{r}\right) \\
& \gamma_{\theta}=\frac{1}{2}\left(\tilde{a}_{\phi r}-\tilde{a}_{r \phi}-\frac{\tilde{b}_{\phi \theta \theta}}{r}\right) \text {, } \\
& \gamma_{\phi}=\frac{1}{2}\left(\tilde{a}_{r \theta}+\frac{\tilde{b}_{r r \theta}}{r}-\tilde{a}_{\theta r}+\frac{\tilde{b}_{\theta \theta \theta}}{r}\right) . \\
& \eta_{r r}=-\frac{1}{2} \tilde{b}_{r \phi \theta}, \\
& \eta_{\theta \theta}=\frac{1}{2} \tilde{b}_{\theta \phi r}, \\
& \eta_{\theta \theta}=\frac{1}{2}\left(\tilde{b}_{\phi r \theta}-\tilde{b}_{\phi \theta r}\right), \\
& \eta_{r \theta}=\eta_{\theta r}=\frac{1}{4}\left(\tilde{b}_{r \phi r}-\tilde{b}_{\theta \phi \theta}\right), \\
& \eta_{r \phi}=\eta_{\phi r}=\frac{1}{4}\left(\tilde{b}_{r r \theta}-\tilde{b}_{\phi \phi \theta}-\tilde{b}_{r \theta r}\right) \text {, } \\
& \eta_{\theta \phi}=\eta_{\phi \theta}=\frac{1}{4}\left(\tilde{b}_{\theta r \theta}+\tilde{b}_{\phi \phi r}-\tilde{b}_{\theta \theta r}\right) \text {. } \\
& \Delta_{r}=\frac{1}{4}\left(\tilde{b}_{\theta \theta r}-\tilde{b}_{\theta r \theta}+\tilde{b}_{\phi \phi r}\right), \\
& \Delta_{\theta}=\frac{1}{4}\left(\tilde{b}_{r r \theta}-\tilde{b}_{r \theta r}+\tilde{b}_{\phi \phi \theta}\right), \\
& \Delta_{\phi}=-\frac{1}{4}\left(\tilde{b}_{r \phi r}+\tilde{b}_{\theta \phi \theta}\right) \text {. }
\end{aligned}
$$


14 Dhang, Bendre, Sharma, \& Subramanian

$$
\begin{array}{rll}
\kappa_{i r r} & = & -\tilde{b}_{i r r}, \\
\kappa_{i \theta \theta}= & -\tilde{b}_{i \theta \theta}, \\
\kappa_{i \phi \phi}= & 0, \\
\kappa_{i r \theta}= & \kappa_{i \theta r}= & -\frac{1}{2}\left(\tilde{b}_{i r \theta}+\tilde{b}_{i \theta r}\right), \\
\kappa_{i r \phi}= & \kappa_{i \phi r}= & -\frac{1}{2} \tilde{b}_{i \phi r}, \\
\kappa_{i \theta \phi}= & \kappa_{i \phi \theta}= & -\frac{1}{2} \tilde{b}_{i \phi \theta},
\end{array}
$$

